\title{
Study Based on the Edge Detection Algorithm Improved by Canny
}

\author{
Zhang Wei and Zhu Sijiao
}

(NanChang Institute of Science \& Technology, Nanchang 330108)

\author{
Keywords: Railway transportation; Foreign invasion limit; Canny arithmetic operators; Open \\ median filter
}

\begin{abstract}
Edge detection is a key technique for detecting foreign objects intrusion on railway rails. For the problem of poor detection of rail image edge, a rail edge detection extraction scheme based on Canny edge detection technology and convergence connection method is proposed in this paper. Through the histogram equalization, the spatial enhancement of original image is achieved, and the details of the image are highlighted, the edge combination of the image is extracted through the Canny edge detection, and the longest two lines in the edge combination satisfying the conditions are extracted by using the convergent continuation method proposed in this paper, and continue to implement it, which can achieve the purpose of rail edge extraction.
\end{abstract}

\section{Introduction}

The edge detection method for foreign invasion is studied. According to the median filter algorithm and switching filter principle, combined with the complex and varied monitoring environment in the scene, an improved switch-based median filter is used to perform the denoising process on the image using a piecewise statistical method. In order to better recover the damaged data, combined with the characteristics of continuous edge trend of the rail, using the inertia principle for edge railing, an improved Canny algorithm is proposed to determine the foreign invasion of the rail, and the problem is tested and analyzed. The experimental results show that the noise suppression ability of this algorithm is much better than that of traditional algorithms. It can be suitable for on-site monitoring and have better continuity and detailed edge performance, improving the automation degree and reliability of the entire monitoring system.

\section{Function of Canny Arithmetic Operators}

When the speed of a train reaches $200 \mathrm{KM} / \mathrm{h}$, even a very small foreign object intruding into the line, it will cause a very serious driving accident. At present, the monitoring method of foreign intrusion in China is mainly video surveillance, that is, cameras are set up in accident-prone areas, and real-time analysis of the collected video is used to determine whether foreign intrusion occurs. This kind of video monitoring requires manual observation and processing, and it consumes a lot of manpower. Therefore, Canny edge detection algorithm is proposed to detect the invasion of foreign objects, which not only improves the degree of automation of the entire monitoring process, but also improves the reliability of the entire system.

Canny defines three criteria for edge detection: SNR criteria, positioning accuracy criteria, and single-edge response criteria. The best edge detection operator is proposed, that is, the Canny edge detection operator. The Canny arithmetic operator has a good detection effect and becomes a standard for evaluating edge detection methods. At the scene of foreign intrusion monitoring, factors such as illumination and weather all affect the quality of the picture, resulting in noise in the image and blurring the detected rail edges. Therefore, according to the on-site monitoring environment, the traditional Canny algorithm should be improved with the characteristics of the Canny algorithm, so that the improved algorithm is more coincident with the practical application. 


\section{Traditional Canny Edge Detection Algorithm Steps}

1. Image smoothing(denoising)

No edge detection algorithm can work well on unprocessed raw data, so the first step is to convolve the original data with a Gaussian mask. The resulting image is slightly blurry compared to the original image. In this way, a single pixel noise becomes almost insignificant on a Gaussian-smoothed image. Equation 1 is a 5 X5 Gaussian filter (Gaussian kernel, standard deviation Idelta $=1.4$ ), where $\mathrm{A}$ is the original image and $\mathrm{B}$ is the smoothed image.

$$
\mathbf{B}=\frac{1}{159}\left[\begin{array}{ccccc}
2 & 4 & 5 & 4 & 2 \\
4 & 9 & 12 & 9 & 4 \\
5 & 12 & 15 & 12 & 5 \\
4 & 9 & 12 & 9 & 4 \\
2 & 4 & 5 & 4 & 2
\end{array}\right] * \mathbf{A}
$$

2. Finding the intensity gradient in the image

The basic idea of Canny algorithm is to find the position where the gray intensity changes the most in a picture. Changing the most is the gradient direction. The gradient of each pixel in the smoothed image can be obtained by the Sobel operator (a convolution operation) (opencv has an encapsulated function to find the $\mathrm{n}$-th derivative of each pixel in the image).

3. Non-maximum suppression

The purpose of this step is to sharpen the blurred boundaries. Popular speaking, the maximum value of gradient intensity at each pixel is preserved, while other values are deleted.

4. Double Thresholding

After non-maximal suppression, there are still many noise points in the image. The Canny algorithm uses a technique called double thresholding. In other words, an upper bound and a lower bound of threshold (usually specified by someone in opencv) are defined. If a pixel in the image is larger than the upper bound of the threshold, it is considered to be a edge (called a strong edge). If the pixel is smaller than the lower bound of the threshold, it isn't considered as anedge inevitably. If the pixel is between the two bound, it is considered as an alternative (called a weak edge) and needs further processing.

5. Using trailing edge railing

The weak edge connected with the strong edge is considered to be the edge, and the other weak edge is suppressed.

\section{Improving Canny Algorithm}

1. Improving median filtering algorithm

Using the method of segmented statistics, first for a pixel P, determine the $(2 \mathrm{~N}+1) \times(2 \mathrm{~N}+1)$ field with the $\mathrm{P}$ point as the center point; here the field is called the window; sort the pixels in the window according to the gray level, and then the sorted pixel values are averagely divided into $\mathrm{N}+2$ segments, each segment contains $\mathrm{N}+2$ pixels, and the average of $\mathrm{N}+2$ pixel values in the middle segment is taken as the new value of P-point pixel gradation. The average value of $\mathrm{N}+2$ pixel gray values in the middle of field image determines the output pixels of the improved algorithm. Due to the correlation among the data points in the window, the isolated noise points can be further eliminated, and the damaged data can also be well restored. Move the window in the image until all pixels have been processed. Experiments show that the improved median filter algorithm performs better in detail protection of images and improves the accuracy of on-site monitoring.

2. Principle of switching filtering

The algorithm processed with switching filtering divides all pixels into a signal $\mathrm{S}$ that maintains the original value and a noise $\mathrm{N}$ that needs to be processed. The algorithm principle is shown in FIG.1. 


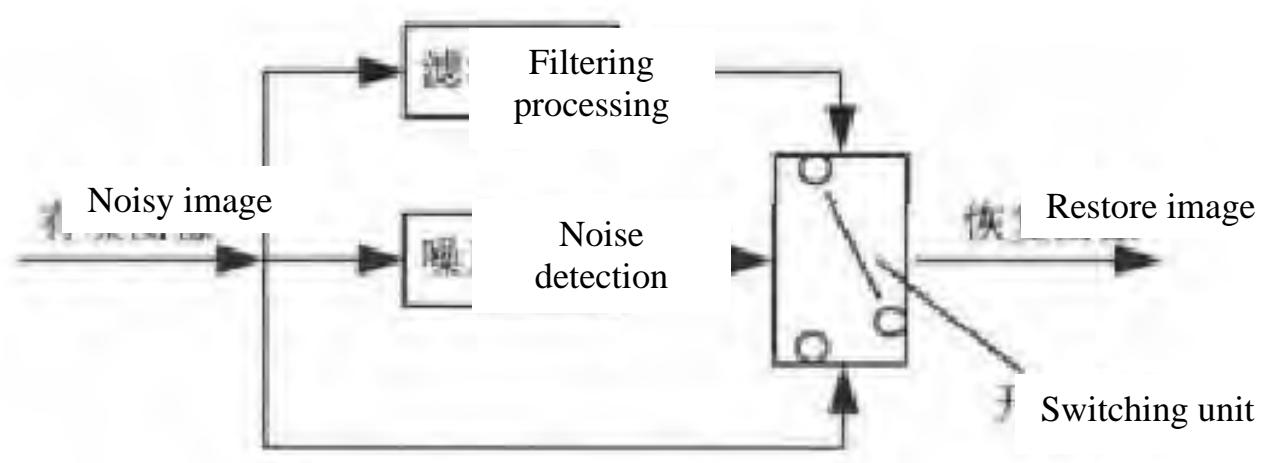

Fig.1 Structure of switching filtering

The noise detector can determine noise to control the switching unit. If noise detector determines that the pixel is not a noise point, the point is directly output without any processing; if it determines that the pixel is a noise point, the switch and the filter processing unit are connected and the point is filtered.

3. Switch denoising based on improved median filtering algorithm

It is a major drawback of traditional median filters to apply a unified approach to all pixels, which can cause image blurring and reduce the accuracy of detection. The switch is used in the filtering process, which can effectively distinguish between signal points and noise points to maximize the detection efficiency and detection accuracy.

The algorithm is also very capable of detail protection for the image, especially having very good detection effect for impact noise, so it'svery suitable for the improved Canny edge detection. Denoising algorithm steps: (1) Move the (3X3) filter window in the image, and overlap the center position of the filter window with the position of a pixel $\mathrm{P}_{0}$ in the image; (2) Read the gray value corresponding to the pixel $\mathrm{P}_{0}$ at this time; (3) Use the max-min operator to perform noise detection on the pixel $\mathrm{P}_{0}$; (4) If the gray value corresponding to the $\mathrm{P}_{0}$ point is not a local extremum, then it's determined that the point is not noise Point, and the original value of the point will be output; (5) If the gray value corresponding to the $\mathrm{P}_{0}$ point is a local extremum, it is determined that the point is a noise point, and this point is processed by the improved median filter algorithm; (6) Process other pixels until they are all processed by using the steps above.
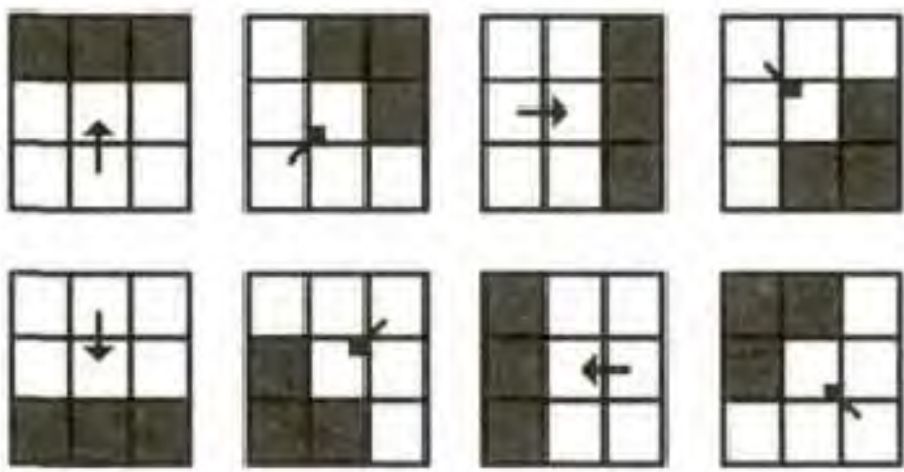

Fig. 2 The direction situation of the edge in $3 \times 3$ window

\section{Conclusion}

The edge detection of the rail pictures is the core of foreign object detection of rail. Only by accurately detecting the dual rails of the line, can the area of obstacle monitoring be determined. After many experiments, the experimental results are obtained: compare with the traditional Canny algorithm, the edge detected by the improved algorithm has better continuity and detailed edge performance, and the noise suppression ability is far better than the traditional algorithm, and more important, it shows strong reliability in on-site monitoring and improves the automation of the 
entire monitoring system.

Fund project: Science and technology project project of NanChang Institute of Science \& Technology (No.GJKJ-16-19)

\section{References}

[1] WangWQ, Ding H F, Xiao X J, et al. The Improved Algorithm of Edge Detection Based on Canny Operator[J]. Advanced Materials Research, 2011, 179-180:97-102.

[2] Zhang B, Sai-Xian H E. Improved Edge-Detection Method Based On Canny Algorithm[J]. Infrared Technology, 2006, 28(3):165-169.

[3] Chai J H, Ying J. Improved Edge-detection Method Based on Canny Algorithm[J]. Video Engineering, 2008.

[4] Niu S, Yang J, Wang S, et al. Improvement and parallel implementation of canny edge detection algorithm based on GPU[J]. 2011:641-644.

[5] Niu S, Yang J, Wang S, et al. Improvement and parallel implementation of canny edge detection algorithm based on GPU[C]// IEEE, International Conference on Asic. IEEE, 2012:641-644.

[6] Tan X. Research on Image Edge Detection based on Improved Canny Algorithm[J]. Journal of Convergence Information Technology, 2013, 8(6):149-156.

[7] Biswas R, Sil J. An Improved Canny Edge Detection Algorithm Based on Type-2 Fuzzy Sets[J]. Procedia Technology, 2012, 4(11):820-824.

[8] Liu C, Zhou J L, Kun H E. Adaptive edge-detection method based on Canny algorithm[J]. Computer Engineering \& Design, 2010, 31(18):4036-4039.

[9] Biswas R, Sil J. An Improved Canny Edge Detection Algorithm Based on Type-2 Fuzzy Sets[C]// International Conference on Computer, Communication, Control and Information Technology. 2012:820-824.

[10] Zhou K, Zhou L, Liu T, et al. Design and Implementation of Real-time Video Edge Detection System Based on Improvement of Canny Algorithm on FPGA[J]. Computer Measurement \& Control, 2016.

[11] Zhang X Y, Zhao R Y. An Adaptive Edge-detection Algorithm Based on Canny and Its Performance Evaluation[J]. Computer Technology \& Development, 2015.

[12] Chen S, Qiu W, Fang Y, et al. Improvement of edge detection algorithm based on Canny operators[J]. Computer Era, 2014. 